Mirete, A.B., Soro, M. \& Maquilón, J.J. (2015). El fracaso escolar y los enfoques de aprendizaje: medidas para la inclusión educativa. Revista Electrónica Interuniversitaria de Formación del Profesorado, 18 (3), 183-196.

DOI: http://dx.doi.org/10.6018/reifop.18.3.239021

\title{
El fracaso escolar y los enfoques de aprendizaje: medidas para la inclusión educativa
}

\author{
Ana Belén Mirete Ruiz*, Marta Soro Bernal*, Javier J. Maquilón Sánchez** \\ anabelen.mirete@um.es, marta.s.b@um.es, jimaqui@um.es \\ *Departamento de Didáctica y Organización Escolar, Universidad de Murcia \\ **Departamento de Métodos de Investigación y Diagnóstico en Educación, Universidad de \\ Murcia
}

\section{RESUMEN}

El índice de fracaso escolar existente actualmente en España resulta muy preocupante a pesar de las numerosas reformas educativas que se han llevado a cabo en los últimos años.

Para analizar los factores motivacionales y de estrategias de aprendizaje que pueden estar incidiendo en la calidad del aprendizaje de los estudiantes se ha realizado una investigación con 281 estudiantes de Educación Secundaria Obligatoria a los que se ha aplicado el Cuestionario sobre Procesos de Aprendizaje (CPA). También se les ha planteado una serie de cuestiones sobre aspectos relacionados con la inclusión, los factores que pueden estar influyendo en el fracaso escolar y las medidas de mejora del rendimiento académico aplicadas por los estudiantes según sus enfoques de aprendizaje.

Con ello se ha podido identificar las características de los aprendizajes de este grupo de estudiantes y se han planteado una serie de propuestas de mejora que de ser aplicadas podrían repercutir en la calidad de sus aprendizajes, en la mejora de su rendimiento académico y en minimizar los nefastos efectos del fracaso escolar en este nivel educativo.

\section{Palabras clave}

Fracaso escolar, enfoques de aprendizaje, rendimiento académico, inclusión educativa

Contacto:

Ana Belén Mirete Ruiz. Departamento de Didáctica y Organización Escolar. Facultad de Educación. Campus de Espinardo, 30100, Murcia. 


\section{School failure and approaches to learning: measures for the educational inclusion}

\section{ABSTRACT}

In spite of the reforms that have been carried out in the past few years, the current school failure rate in Spain continues to be a major concern.

In order to analyse the motivational and strategical factors that may have had an impact on the quality of learning, a research has been accomplished. 281 students of Secondary Education have participated in this study, fulfilling the Study Process Questionnaire (LPQ). Students have also been asked about inclusion, the factors that may influence school failure as well as about the measures to improve the academic performance depending on their learning approach.

The aforementioned study led us to identify the learning characteristics of the students and several improving measures have been proposed. Those measures could have a positive impact on the quality of their learning, increase their academic performance as well as minimize the effects of school failure in this stage.

\section{Key words}

School failure, approaches to learning, academic achievement/performance, educational inclusion.

\section{INTRODUCCIÓN}

La orientación de las investigaciones acerca del aprendizaje de los alumnos sufrió un cambio radical a partir de la década de los 70. Con anterioridad, el rendimiento académico se relacionaba, sobre todo, con factores como el cociente intelectual, la situación socioeconómica, los conocimientos previos, la calidad y formación de los docentes, etc. En cambio, en las últimas décadas, se ha modificado esta percepción, existiendo actualmente gran cantidad de información sobre los modos preferentes y los recursos que los estudiantes emplean para aprender.

Marton y Säljö emplearon inicialmente el concepto de enfoques de aprendizaje (approaches to learning) para referirse a la adaptación de estrategias de estudio que llevan a cabo los alumnos para afrontar distintas tareas a lo largo de su vida como estudiantes. Estos autores realizaron una investigación que consistió en dar un artículo a alumnos universitarios para que lo comprendieran. Tras analizar los resultados, obtuvieron patrones de comportamiento definidos, estableciendo la distinción entre enfoque profundo y enfoque superficial, términos utilizados para describir formas de aprender de una tarea. Los alumnos que utilizaron un enfoque profundo trataron de buscar el significado general del artículo; aquellos que utilizaron un enfoque superficial memorizaron cierta información, sin obtener una visión global de lo que se decía en él.

Entwistle y Ramsden desarrollaron un modelo de aprendizaje y un cuestionario cuya finalidad era facilitar la obtención de una medida de los enfoques utilizados por los alumnos: el Approaches to Study Inventory (ASI, Inventario de Enfoques de Estudio).

El análisis de las respuestas de los sujetos estableció tres dimensiones (Hernández-Pina, 1993): el enfoque superficial, el profundo y el estratégico. 
A continuación, en la tabla 1, se muestra cómo se caracteriza cada dimensión.

Tabla 1

Características de los enfoques de aprendizaje de Entwistle (Adaptado de Hernández Pina (1993))

\begin{tabular}{|c|c|c|c|c|}
\hline & Motivación & Intención & Procesos & Resultados \\
\hline $\begin{array}{l}\text { Enfoque } \\
\text { superficial }\end{array}$ & $\begin{array}{l}\text { Cumplir con el } \\
\text { curso. } \\
\text { Miedo al } \\
\text { fracaso. }\end{array}$ & $\begin{array}{l}\text { Cumplir con los } \\
\text { requisitos de la } \\
\text { evaluación } \\
\text { mediante la } \\
\text { reproducción. }\end{array}$ & $\begin{array}{l}\text { Aprender de } \\
\text { memoria, por } \\
\text { repetición, } \\
\text { hechos o ideas } \\
\text { apenas } \\
\text { interrelaciones. }\end{array}$ & $\begin{array}{l}\text { Nivel de } \\
\text { comprensión } \\
\text { nulo o } \\
\text { superficial. }\end{array}$ \\
\hline $\begin{array}{l}\text { Enfoque } \\
\text { profundo }\end{array}$ & $\begin{array}{l}\text { Buscar una } \\
\text { relevancia } \\
\text { vocacional. } \\
\text { Interés por la } \\
\text { materia. }\end{array}$ & $\begin{array}{l}\text { Conseguir que } \\
\text { todo tenga una } \\
\text { significación } \\
\text { personal. }\end{array}$ & $\begin{array}{l}\text { Aprendizaje por } \\
\text { comprensión, } \\
\text { por operación. }\end{array}$ & $\begin{array}{l}\text { Nivel de } \\
\text { comprensión } \\
\text { profundo si se } \\
\text { integran } \\
\text { principios y } \\
\text { hechos y si se } \\
\text { buscan } \\
\text { argumentos. }\end{array}$ \\
\hline $\begin{array}{c}\text { Enfoque } \\
\text { estratégico }\end{array}$ & $\begin{array}{l}\text { Conseguir notas } \\
\text { elevadas. } \\
\text { Competir con } \\
\text { los demás. }\end{array}$ & $\begin{array}{l}\text { Obtener el éxito } \\
\text { por los medios } \\
\text { que sean. }\end{array}$ & $\begin{array}{l}\text { Aprendizaje por } \\
\text { memorización, } \\
\text { por comprensión } \\
\text { o por operación. }\end{array}$ & $\begin{array}{l}\text { En función de las } \\
\text { características } \\
\text { del curso } \\
\text { (objetivos, } \\
\text { métodos de } \\
\text { evaluación...). }\end{array}$ \\
\hline
\end{tabular}

Los enfoques de aprendizaje descritos por Biggs se generan dentro de un modelo, denominado Modelo $3 \mathrm{P}$ de aprendizaje escolar, el cual es un sistema integrado por tres componentes: Presagio, Proceso y Producto. Estas tres variables tienden al equilibrio, por lo que un cambio en alguna de ellas repercutiría en todo el sistema.

La variable presagio incluye las características individuales de profesores y alumnos implicados en el proceso de enseñanza-aprendizaje. La variable proceso se refiere al proceso de aprendizaje, integrado por la motivación y las estrategias que emplean los estudiantes en la dinámica de enseñanza-aprendizaje. Dichas estrategias se agrupan en función de tres enfoques (superficial, profundo y de alto rendimiento) (Maquilón, 2001). La variable producto es el resultado de aprendizaje, determinado por los enfoques adoptados por los alumnos al aprender. Esta variable está condicionada por la interacción entre las dos variables anteriores (presagio y proceso).

Los enfoques de aprendizaje se constituyen como un conjunto de intenciones y estrategias que orientan y condicionan la conducta del alumno durante el proceso de aprendizaje. Por tanto, dentro de los enfoques de aprendizaje se diferencian dos subescalas: motivo y estrategia. Los motivos de aprendizaje que guían a un estudiante están relacionados con los 
resultados académicos que pretende conseguir y mantienen una relación directa con el componente cognitivo de los estudiantes. Se pueden establecer tres niveles básicos: motivación intrínseca, motivación extrínseca y necesidad de logro. Por otro lado, la aplicación de estrategias de aprendizaje es necesaria para llevar a cabo las tareas de estudio. La selección de la estrategia específica depende de la naturaleza de la tarea, y su empleo permite alcanzar mejores logros, incluso en aquellas situaciones para las que un cierto estilo de aprendizaje podría no ser el más adecuado (López-Vargas, HederichMartínez y Camargo-Uribe, 2011).

En cuanto a la motivación, ésta se constituye como el motor del aprendizaje, entrando a formar parte activa del actuar del estudiante. Su presencia o no, no depende solamente de las características personales del alumno, sino que también influyen otros aspectos. Según De La Fuente y Justicia (2004), la motivación es una variable muy importante ya que no hay un modelo de aprendizaje que no incorpore una teoría de la motivación.

Las variables personales son las que más van a determinar la motivación escolar, siendo necesario, para incidir en la motivación, hacer referencia al autoconcepto, los patrones de atribución causal y las metas de aprendizaje.

Algunos autores hacen una distinción entre metas de aprendizaje y metas de ejecución o rendimiento (Elliot y Dweck, 1988), dando lugar a dos tipos de motivación: intrínseca y extrínseca.

La motivación intrínseca es aquella que proviene del propio sujeto; la tarea en sí es la recompensa. Cuando el alumno experimenta este tipo de motivación, muestra gran curiosidad e interés por la tarea, por lo que está asociada con grandes logros educativos por parte de los estudiantes. Está muy ligada al enfoque profundo de aprendizaje y a una orientación competitiva de autosuperación del enfoque de alto rendimiento (Maquilón, 2001). La motivación extrínseca es aquella que procede de fuera y conduce a la ejecución de la tarea. Viene marcada por motivos sociales y la motivación de logro, por lo que empuja a la consecución de un objetivo reconocido socialmente.

La motivación que tenga un alumno mantiene una relación directa con los enfoques de aprendizaje, y la adopción de uno u otro dependerá de la intención que tenga el estudiante hacia las tareas escolares.

El tipo de motivación podría estar relacionado con la utilización de determinadas estrategias de aprendizaje y, por consiguiente, con el enfoque usado por el estudiante. Todos estos factores van a incidir en la calidad de los resultados académicos. (Biggs, 1978a).

También el rendimiento escolar está en relación con los enfoques de aprendizaje que emplee el estudiante. De Natale (1990) define el rendimiento académico como el conjunto de habilidades, destrezas, hábitos, ideales, aspiraciones, intereses, inquietudes y realizaciones que aplica el estudiante para aprender. Según Manzano (2007), el rendimiento académico es el resultado del esfuerzo y la capacidad de trabajo que ha tenido el estudiante durante el proceso de enseñanza-aprendizaje.

Estudios previos (Hernández-Pina, García \& Maquilón, 2005, Mirete, 2014) pone de manifiesto que la calidad del aprendizaje de los estudiantes está relacionada con la calidad de enseñanza del profesor. Por tanto, los profesores que son conscientes de sus enfoques de enseñanza (intenciones y estrategias) y los emplean adecuadamente, obtienen mejores resultados académicos con sus estudiantes (Maquilón, 2010). Este uso adecuado de los enfoques de enseñanza debe interpretarse como la correcta relación entre intenciones y estrategias. Es decir, si un profesor tiene la intención de transmitir información a sus alumnos, debe emplear las estrategias acordes a dichas intenciones y evaluar acorde a las 
mismas. Si por el contrario, el profesor tiene la intención de que sus estudiantes construyan su propio conocimiento, debe emplear las estrategias adecuadas a estas intenciones. Así, las situaciones de enseñanza-aprendizaje serán más eficaces y efectivas.

Existe un vínculo entre rendimiento académico y fracaso escolar, ya que a medida que aumenta el rendimiento disminuye el fracaso, apareciendo este último cuando no se alcanzan unos mínimos establecidos previamente. (Maquilón y Hernández-Pina, 2011).

No existe una definición universal y unánimemente aceptada del concepto "fracaso escolar". Marchesi (2003) asocia el fracaso escolar con aquellos alumnos que al término de la educación obligatoria no se sienten interesados en realizar nuevos aprendizajes o no se sienten capaces para ello. Los alumnos que fracasan serían aquellos que, al finalizar su permanencia en la escuela, no han alcanzado los conocimientos y habilidades que se consideran necesarios para manejarse de forma satisfactoria en la vida social y laboral o proseguir sus estudios.

Según Calero, Choi y Waisgrais, (2009: 225) el fracaso escolar es entendido como la "proporción de individuos que no consiguen concluir los estudios obligatorios, es uno de los principales problemas del sistema educativo español".

Los estudios realizados para tratar de identificar las causas asociadas al fracaso escolar son numerosos. Aunque existe gran cantidad de factores que pueden influir en esta problemática, nos centraremos aquí en el centro educativo, el profesorado, el contexto familiar y en el propio alumnado.

El centro escolar puede tener una influencia en la problemática del fracaso escolar. Marchesi y Pérez (2003) destacan que la eficacia y el éxito de las escuelas dependen de los rasgos que definen el centro escolar, así como del esfuerzo compartido de toda la comunidad educativa.

Otro aspecto a tener en cuenta a la hora de analizar el éxito o fracaso escolar es el profesorado, considerado un elemento clave en el desarrollo personal y académico del alumno, ya que la figura del profesor se erige como una referencia para los estudiantes. En un estudio realizado por Pérez-Díaz, Rodríguez y Sánchez (2001), se obtuvo la conclusión de que existía una relación directa entre los resultados académicos de los estudiantes y la percepción de valía profesional que tenían sus padres respecto a los docentes. También las expectativas que los profesores tienen de sus alumnos influyen en los resultados académicos de éstos.

Factores como la clase social y el nivel de estudios de los padres pueden ser condicionantes del fracaso escolar. No obstante, en una investigación realizada por Balzano (2002), se confirma que, con independencia de la clase social, los padres y madres atribuyen el fracaso escolar a factores relacionados con lo que sucede en la propia familia, factores propios del estudiante y factores escolares.

En relación al alumnado, se deben analizar aspectos como los rasgos de personalidad, el nivel de autoestima, las estrategias de aprendizaje que emplea o la relación que mantiene con sus iguales para poder diseñar la metodología de aprendizaje que más se adapte a sus características, reforzando sus puntos fuertes y mejorando los más débiles.

En cualquier caso, la problemática del fracaso escolar tiene efectos en toda la colectividad. En un entorno cada vez más competitivo, las consecuencias son graves, ya que los alumnos que no han alcanzado unos mínimos, encuentran mayores dificultades para incorporarse al mercado laboral, y además ocupando puestos menos cualificados, menor remunerados y con pocas posibilidades de promoción. Por otro lado, cada vez se exigen trabajadores más cualificados y con mayor grado de flexibilidad para adaptarse a un entorno altamente 
cambiante, por lo que la motivación y el esfuerzo de los escolares tienen un papel decisivo en el proceso de aprendizaje. En definitiva, el sistema educativo español viene produciendo en los últimos años unos resultados que, comparados con otros países de la UE, son bastante mejorables. Según datos presentados por la Comisión Europea, el índice de abandono escolar que presenta nuestro país es del 23.5\% (año 2013), el mayor de toda la UE.

Ante esta situación, se ha realizado una investigación que tiene por objetivo analizar los enfoques de aprendizaje de los estudiantes y las características del fracaso escolar, así como la relación de éstos con el rendimiento académico, identificando las medidas de mejora propuestas por los propios estudiantes de $3^{\circ}$ y $4^{\circ}$ de E.S.O.

Para dar respuesta a este objetivo general se han planteado cuatro objetivos específicos que son:

Describir enfoques de aprendizaje de los estudiantes a nivel global y según el curso.

Identificar los factores determinantes del fracaso escolar desde la perspectiva de los estudiantes.

Analizar el rendimiento académico según el enfoque de aprendizaje.

Valorar las medidas de mejora del rendimiento académico aplicadas por los estudiantes según sus enfoques de aprendizaje.

\section{MÉTODO}

\section{Participantes y contexto}

En esta investigación participaron 281 estudiantes de tercer y cuarto curso de E.S.O.

La distribución de los mismos según el curso se detalla en la tabla 2.

Tabla 2.

Participantes según el curso

\begin{tabular}{|c|c|c|}
\hline CURSO & FRECUENCIA & PORCENTAJE \\
\hline $3^{\circ}$ E.S.O & 140 & $49.8 \%$ \\
\hline $4^{\circ}$ E.S.O & 141 & $50.2 \%$ \\
\hline TOTAL & 281 & $100 \%$ \\
\hline
\end{tabular}

El diseño seguido en la investigación es cuantitativo tipo encuesta, ya que a los participantes se les ha aplicado un cuestionario con preguntas que aportan información para responder al problema de investigación en general y, a los objetivos, en particular.

El instrumento empleado es el Cuestionario sobre Procesos de Aprendizaje (CPA), adaptado y traducido por la Dra. Hernández-Pina (1994) del Study Process Questionnaire (Biggs, 1987b). Este cuestionario está compuesto por 36 ítems que reproducen un modelo jerárquico de seis subescalas, tres motivacionales y tres estratégicas, configurando finalmente, de acuerdo con el modelo teórico original, los tres enfoques de aprendizaje.

A fin de poder dar respuesta a los objetivos de esta investigación, al cuestionario CPA se le han añadido otras preguntas que recaban información sobre las calificaciones de 
determinadas asignaturas, circunstancias que han llevado al alumno a su situación escolar y medidas que están llevando a cabo para mejorar.

\section{ANÁLISIS Y RESULTADOS}

En el primero de los objetivos se describen los enfoques de aprendizaje de los estudiantes a nivel global y según el curso. Como puede observarse en la tabla 3, las medias más altas son las del enfoque profundo (36.15) y las de alto rendimiento (36.20), por lo que el grado de afianzamiento de estos enfoques es mayor que en el enfoque superficial, interpretándose que los estudiantes que emplean un enfoque (predominantemente) profundo lo hace con mayor intensidad y convicción que el que emplea un enfoque superficial. Igualmente $84.7 \%$ de los estudiantes conoce y utiliza estrategias y motivaciones adecuadas de cara al estudio y el aprendizaje.

Tabla 3.

Media, desviación típica y porcentaje de los enfoques de aprendizaje

\begin{tabular}{|c|c|c|c|}
\cline { 2 - 4 } \multicolumn{1}{c|}{} & SA & DA & AA \\
\hline Media & 31.75 & 36.15 & 36.20 \\
\hline Desviación típica & 5.983 & 7.029 & 8.005 \\
\hline Porcentaje & $15.3 \%$ & $36.7 \%$ & $48 \%$ \\
\hline
\end{tabular}

Al analizar los enfoques de aprendizaje según el curso (Tabla 4) se encontró que los estudiantes de $3^{\circ}$ de E.S.O. tuvieron la media más en el enfoque profundo (36.01), siendo éste el más afianzado, seguido de los valores de alto rendimiento (34.78) y de enfoque superficial (32.59).

Por otro lado, los estudiantes de $4^{\circ}$ de E.S.O., obtuvieron la media más alta en el enfoque de alto rendimiento (37.61), seguida de la media de enfoque profundo (36.29) y el enfoque superficial (30.92).

Si comparamos los datos entre ambos cursos, apreciamos que los alumnos de $4^{\circ}$ tienen más afianzado el enfoque profundo y de alto rendimiento que los alumnos de $3^{\circ}$, mientras que éstos presentan un mayor nivel de afianzamiento del enfoque superficial. Interpretamos por ello que los estudiantes de $4^{\circ}$ están más motivados y utilizan mejores estrategias, encontrando casi un $10 \%$ más de empleo del enfoque de alto rendimiento que en los alumnos de $3^{\circ}$.

Tabla 4.

Descriptivo de los enfoques de aprendizaje según el curso

\begin{tabular}{|c|c|c|c|c|}
\hline \multicolumn{2}{|c|}{ GLOBAL } & \multicolumn{3}{c|}{ Media / Desviación típica / Porcentaje } \\
\hline CURSO & N & SA & DA & AA \\
\hline $3^{\circ}$ ESO & 140 & $32.59 / 6.134 / 20.0$ & $36.01 / 6.600 / 38.6$ & $34.78 / 8.158 / 41.4$ \\
\hline $4^{\circ}$ ESO & 141 & $30.92 / 5.731 / 10.6$ & $36.29 / 7.451 / 34.8$ & $37.61 / 7.620 / 54.6$ \\
\hline
\end{tabular}


En el segundo de los objetivos se identificaron los factores determinantes del fracaso escolar desde la perspectiva de los estudiantes. Se establecieron las siguientes categorías en función de las respuestas de los participantes: (1) Desmotivación/desinterés; (2) Dificultad con la asignatura; (3) Problemas con el profesor/a; (4) Problemas personales y/o familiares; (5) Desmotivación/desinterés y dificultad con la asignatura; (6) Desmotivación/desinterés y problemas con el profesor/a; (7) Desmotivación/desinterés y problemas personales y/o familiares; (8) Dificultad con la asignatura y problemas con el profesor/a; (9) Dificultad con la asignatura y problemas personales y/o familiares; (10) Problemas con el profesor/a y problemas personales/familiares; (11) Otros.

Los resultados obtenidos (Tabla 5) indican que la causa principal que lleva a un estudiante a tener un bajo rendimiento en determinadas materias es la dificultad a la hora de abordar la asignatura (24.9\%), seguida de la desmotivación/desinterés (23.8\%). También la conjunción de ambas causas tiene un peso importante (14.9\%), pues existe una relación directa entre ellas. Aquellas asignaturas que resulten más difíciles para un alumno requerirán un mayor esfuerzo y dedicación. Sin embargo, si no trabajan lo suficiente, pueden quedar desenganchados y esto podría llevarles a la desmotivación. Por otro lado, cabe destacar el bajo porcentaje de la respuesta "Problemas con el profesor/a" (3.20\%), lo cual demuestra que la relación profesor-alumno es, en general, buena. Resulta llamativo la escasa elección de la respuesta "Problemas personales y/o familiares" (1.10\%), dado que la adolescencia es una etapa de grandes cambios y descubrimientos que, en ocasiones, conlleva ciertas dificultades.

Tabla 5.

Factores que influyen en el fracaso escolar

\begin{tabular}{|c|c|c|c|c|c|c|}
\hline GLOBAL & 1 & 2 & 3 & 4 & 5 & 6 \\
\hline Frecuencia & 67 & 70 & 9 & 3 & 42 & 7 \\
\hline Porcentaje & 23.8 & 24.9 & 3.2 & 1.1 & 14.9 & 2.5 \\
\hline
\end{tabular}

\begin{tabular}{|c|c|c|c|c|c|c|}
\hline GLOBAL & 7 & 8 & 9 & 10 & 11 & Perdidos \\
\hline Frecuencia & 17 & 12 & 8 & 2 & 36 & 8 \\
\hline Porcentaje & 6.0 & 4.3 & 2.8 & 0.7 & 12.8 & 2.8 \\
\hline
\end{tabular}

En el tercer objetivo se analizó el rendimiento académico de los estudiantes según sus enfoques de aprendizaje. Este rendimiento se valoró en función de las calificaciones obtenidas en las asignaturas de lengua, matemáticas e idioma, calculando la nota media de la primera y la segunda evaluación de dichas asignaturas.

En cuanto a la asignatura de Lengua (Tabla 6) se destaca que más de la mitad de alumnos que suspende esta asignatura (65.7\%) adopta un enfoque de alto rendimiento, lo cual resulta llamativo porque habitualmente los estudiantes que tienen un bajo rendimiento académico emplean un enfoque superficial. Para aquellos alumnos que obtienen sobresaliente en la materia de lengua, existe una mayor proporción de estudiantes con enfoque superficial (35.3\%) que de alto rendimiento (11.8\%). Sin embargo, la mayoría emplea predominantemente el enfoque profundo (52.9\%). Esto puede indicar que los procedimientos de evaluación de esta asignatura están muy relacionados con el aprendizaje 
memorístico, permitiendo que estudiantes con un enfoque poco adecuado obtengan buenos resultados académicos.

En cambio, los estudiantes estratégicos van desapareciendo cuando las calificaciones son más altas, Ilegando a ser solo dos cuando la calificación en Lengua es de Sobresaliente.

Tabla 6.

Enfoques de aprendizaje y calificaciones obtenidas en la asignatura de lengua.

\begin{tabular}{|c|c|c|c|c|}
\cline { 2 - 5 } \multicolumn{1}{c|}{} & \multicolumn{4}{c|}{ Frecuencia / Porcentaje } \\
\hline Lengua & SA & DA & AA & TOTAL \\
\hline Suspenso & $6 / 5.7 \%$ & $30 / 28.6 \%$ & $69 / 65.7 \%$ & $100 \%$ \\
\hline Aprobado & $4 / 9.5 \%$ & $17 / 40.5 \%$ & $21 / 50.0 \%$ & $100 \%$ \\
\hline Bien & $6 / 21.4 \%$ & $10 / 35.7 \%$ & $12 / 42.9 \%$ & $100 \%$ \\
\hline Notable & $21 / 23.6 \%$ & $37 / 41.6 \%$ & $31 / 34.8 \%$ & $100 \%$ \\
\hline Sobresaliente & $6 / 35.3 \%$ & $9 / 52.9 \%$ & $2 / 11.8 \%$ & $100 \%$ \\
\hline
\end{tabular}

Al analizar la asignatura de Matemáticas (Tabla 7), se observó que mayoritariamente es empleado el enfoque profundo y el de alto rendimiento por aquellos estudiantes cuyas calificaciones están entre bien y notable. De entre los que suspenden esta asignatura, el $63.4 \%$ adopta un enfoque de alto rendimiento, mientras que el $7.1 \%$ emplea un enfoque superficial. Para aquellos que obtienen la calificación Bien, predomina el enfoque profundo (46.7\%), seguido del alto rendimiento (40.0\%) y, en menor medida, del enfoque superficial (13.3\%). Los alumnos que obtuvieron la calificación de notable son prioritariamente profundos (41.4\%), y en menor medida superficiales (30.0\%) y de alto rendimiento $(28.6 \%)$. Finalmente, para la calificación de Sobresaliente, el porcentaje de alumnos con enfoque superficial y de alto rendimiento es el mismo (37.5\%), mientras que el $25.0 \%$ adopta un enfoque profundo.

Estos datos resultan llamativos, puesto que los alumnos que suspenden u obtienen un aprobado tienen más afianzado el enfoque de alto rendimiento que el superficial. Esto es así porque un alumno superficial puede confundir lo que hace con lo que le gustaría ser, es decir, que suele tener una motivación de alto rendimiento, equivalente a lo que le gustaría ser y que sabe que difícilmente conseguirá. 
Tabla 7.

Enfoques de aprendizaje y calificaciones obtenidas en la asignatura de matemáticas

\begin{tabular}{|c|c|c|c|c|}
\cline { 2 - 5 } \multicolumn{1}{c|}{} & \multicolumn{4}{c|}{ Frecuencia / Porcentaje } \\
\hline Matemáticas & SA & DA & AA & TOTAL \\
\hline Suspenso & $8 / 7.1 \%$ & $33 / 29.5 \%$ & $71 / 63.4 \%$ & $100 \%$ \\
\hline Aprobado & $3 / 9.7 \%$ & $11 / 35.5 \%$ & $17 / 54.8 \%$ & $100 \%$ \\
\hline Bien & $8 / 13.3 \%$ & $28 / 46.7 \%$ & $24 / 40.0 \%$ & $100 \%$ \\
\hline Notable & $21 / 30.0 \%$ & $29 / 41.4 \%$ & $20 / 28.6 \%$ & $100 \%$ \\
\hline Sobresaliente & $3 / 37.5 \%$ & $2 / 25.0 \%$ & $3 / 37.5 \%$ & $100 \%$ \\
\hline
\end{tabular}

Con la asignatura Inglés (Tabla 8), se mantiene la tendencia detectada en las otras dos asignaturas. Predomina el enfoque de alto rendimiento tanto para los alumnos que suspenden (64.2\%) como para los que obtienen un aprobado (63.6\%). En las calificaciones de Bien y Notable, el enfoque más afianzado es el profundo (55.3\% y $40.0 \%$ respectivamente) y el que menos predomina es el superficial (10.5\% y $23.3 \%$ respectivamente). Finalmente, el porcentaje de alumnos que obtiene un sobresaliente y que emplea un enfoque superficial o de alto rendimiento es el mismo (26.1\%), aunque el más empleado es el enfoque profundo (47.8\%).

Tabla 8.

Enfoques de aprendizaje y calificaciones obtenidas en la asignatura de Inglés

\begin{tabular}{|c|c|c|c|c|}
\cline { 2 - 5 } \multicolumn{1}{c|}{} & \multicolumn{4}{c|}{ Frecuencia / Porcentaje } \\
\hline Idioma & SA & DA & AA & TOTAL \\
\hline Suspenso & $8 / 9.9 \%$ & $21 / 25.9 \%$ & $52 / 64.2 \%$ & $100 \%$ \\
\hline Aprobado & $4 / 8.2 \%$ & $14 / 28.6 \%$ & $31 / 63.6 \%$ & $100 \%$ \\
\hline Bien & $4 / 10.5 \%$ & $21 / 55.3 \%$ & $13 / 34.2 \%$ & $100 \%$ \\
\hline Notable & $21 / 23.3 \%$ & $36 / 40.0 \%$ & $33 / 36.7 \%$ & $100 \%$ \\
\hline Sobresaliente & $6 / 26.1 \%$ & $11 / 47.8 \%$ & $6 / 26.1 \%$ & $100 \%$ \\
\hline
\end{tabular}

En el último objetivo se valoraron las medidas de mejora del rendimiento académico aplicadas por los estudiantes a nivel global y según sus enfoques de aprendizaje,

A partir de los datos obtenidos, se han establecido las siguientes categorías sobre las mejoras propuestas por los estudiantes: (1) Utilización de técnicas de estudio; (2) Clases de apoyo; (3) Planificación del tiempo y las tareas; (4) Auto motivación; (5) Utilización de técnicas de estudio y clases de apoyo; (6) Utilización de técnicas de estudio y planificación del tiempo y las tareas; (7) Utilización de técnicas de estudio y auto motivación; (8) Clases 
de apoyo y planificación del tiempo y las tareas; (9) Clases de apoyo y auto motivación; (10) Planificación del tiempo y las tareas y auto motivación; (11) Otros.

De los datos recogidos en la tabla 9 destacamos que la medida preferente a la que acuden los estudiantes para tratar de mejorar su rendimiento académico es la planificación del tiempo y las tareas (25.3\%). Dedicar las horas necesarias y ser constante con las actividades escolares puede resultar útil para incrementar los resultados, aunque sería más importante que los alumnos mejorasen sus estrategias de estudio. La auto motivación (10.7\%) es otra de las medidas que están aplicando los estudiantes para mejorar; pues nadie sabe mejor que ellos lo que les induce al esfuerzo. La combinación de las dos medidas anteriores es otra buena estrategia de mejora (16.4\%). El 15.7\% utiliza técnicas de estudio en combinación con la planificación del tiempo y las tareas. Establecerse un horario que ayude a crear un hábito de estudio diario favorece la constancia de los estudiantes.

El $5.0 \%$ considera que debe utilizar técnicas de estudio como una de las soluciones para mejorar el rendimiento.

Tabla 9.

Medidas de mejora del rendimiento académico

\begin{tabular}{|c|c|c|c|c|c|c|}
\hline GLOBAL & 1 & 2 & 3 & 4 & 5 & 6 \\
\hline Frecuencia & 14 & 28 & 71 & 30 & 12 & 44 \\
\hline Porcentaje & 5.0 & 10.0 & 25.3 & 10.7 & 4.3 & 15.7 \\
\hline
\end{tabular}

\begin{tabular}{|c|c|c|c|c|c|c|}
\hline GLOBAL & 7 & 8 & 9 & 10 & 11 & Perdidos \\
\hline Frecuencia & 6 & 18 & 6 & 46 & 6 & 0 \\
\hline Porcentaje & 2.1 & 6.4 & 2.1 & 16.4 & 2.1 & 0 \\
\hline
\end{tabular}

En la figura 1 se incluye un síntesis de las medidas llevadas a cabo los estudiantes para mejorar su rendimiento académico según el enfoque de aprendizaje preferente. Se destaca la planificación del tiempo y las tareas para los alumnos con enfoque profundo, seguido de las clases de apoyo. Los alumnos con enfoque de alto rendimiento optan también por planificar el tiempo y las tareas, es decir, conocen las estrategias de estudio pero necesitan planificarse. Finalmente, los alumnos con enfoque superficial también optan por la planificación del tiempo y las tareas y, en menor medida, la auto motivación. También se destaca que la medida para mejorar el rendimiento menos recurrida por los tres grupos de estudiantes es la utilización de técnicas de estudio, lo cual supone un error de percepción por parte de los mismos como se comentará en el apartado conclusiones. 


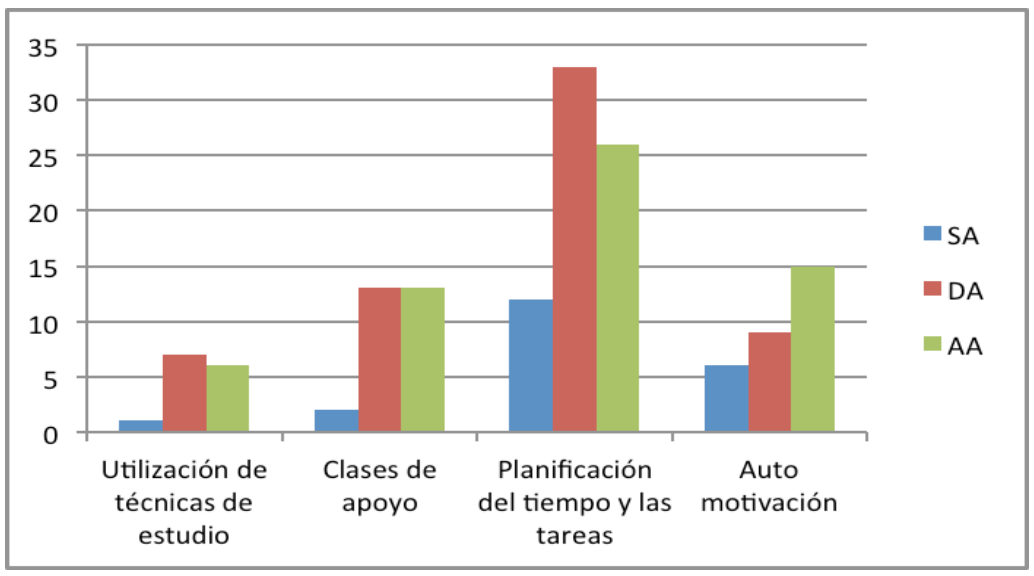

Figura 1. Medidas de mejora del rendimiento según el enfoque de aprendizaje

\section{DISCUSIÓN Y CONCLUSIONES}

De las conclusiones obtenidas en la investigación resaltamos que el enfoque de aprendizaje predominante es el de alto rendimiento, seguido del enfoque profundo, lo cual no coincide con estudios previos (Maquilón y Hernánez Pina, 2011) en el que el enfoque mayoritario fue el profundo seguido del superficial. Esto demuestra que gran parte del alumnado conoce y aplica estrategias de aprendizaje; sin embargo, existe una falta de motivación importante, factor que desemboca en un menor rendimiento académico.

Según el curso académico se observó que los estudiantes de $3^{\circ}$ de E.S.O. tienen más afianzado el enfoque profundo, mientras que los estudiantes de $4^{\circ}$ obtienen una mayor puntuación en el enfoque de alto rendimiento. Esto puede ser debido a que al tener que escoger entre algunas asignaturas (optativas), se han decantado por las que más les gustaban, lo cual hace que estén más motivados e implicados en el aprendizaje escolar. El enfoque superficial es el menos afianzado para los dos cursos lo cual es destacable por el hecho de no existir de un modo generalizado buenos resultados académicos entre los estudiantes.

Respecto a los resultados académicos, se observa un alto porcentaje de estudiantes que a pesar de suspender u obtener unas calificaciones bajas, dice emplear estrategias de alto rendimiento. En cambio, para los alumnos que tienen un rendimiento académico alto, predomina el enfoque superficial. Ante estos resultados, se puede deducir que los estudiantes con bajos resultados no han contestado el cuestionario sobre cómo son ellos realmente, sino cómo les gustaría ser. También el procedimiento de evaluación de cada asignatura estarán relacionado con los resultados académicos porque si los que obtienen buenos resultados afirman emplear enfoque superficial, indica que son evaluados de modo memorístico o escasamente comprensivo por parte del profesorado.

Por otra parte, en cuanto a los factores determinantes del fracaso escolar, encontramos, en primer lugar, la dificultad con la asignatura. Esto puede ser debido a la complejidad de los contenidos que se estudian y también al nivel previo de los estudiantes. Si no está consolidado adecuadamente lo que se estudió en cursos anteriores, resulta más difícil adquirir nuevos conocimientos. El segundo factor que explica el bajo rendimiento de los estudiantes es la falta de motivación y de interés, por lo que deberían fomentarse ambos aspectos en el aula, ya sea modificando la metodología docente o realizando programas específicos para tal fin. Los factores que menos determinan el bajo rendimiento son "Problemas personales y/o familiares" y "Problemas con el profesor/a", lo que demuestra 
que, a nivel general, los estudiantes mantienen una buena relación con su entorno, ya sea familiar o educativo.

Finalmente, los estudiantes aplican como método de mejora de su rendimiento la planificación del tiempo y las tareas, que está estrechamente relacionado con el rendimiento escolar. El trabajo diario favorece la creación de hábitos que hacen más llevaderos el estudio y evitan la acumulación de tareas en el último momento. La segunda medida adoptada por los estudiantes con enfoque superficial y de alto rendimiento es la auto motivación. Estos estudiantes conocen las estrategias de aprendizaje, pero necesitan auto motivarse, poniéndose metas o logros académicos a corto y medio plazo. Los estudiantes con enfoque profundo recurren a clases de apoyo. En contraposición, la utilización de técnicas de estudio es una medida poco empleada por los estudiantes independientemente del enfoque de aprendizaje que empleen. Sin embargo, su utilización puede ayudar a mejorar el rendimiento de los estudiantes.

Si comparamos los resultados obtenidos en esta investigación con estudios previos (Calero, Choi \& Waisgrais, 2009, Maquilón \& Hernández Pina, 2011, Mirete, 2014) se constata que no están en la misma línea. Si bien, se ha constatado que los enfoques de aprendizaje están en relación con el fracaso escolar, aunque en esta investigación no se ha demostrado una relación directa ya que los estudiantes con un enfoque poco recomendable obtienen buenos resultados académicos. Sin embargo, la falta de motivación y unas estrategias de estudio poco adecuadas son previsoras de futuros fracasos escolares.

Por otro lado, los resultados obtenidos muestran que el uso de unas estrategias adecuadas y una motivación intrínseca no siempre está relacionada con las mejores calificaciones académicas, lo cual indica la necesidad de profundizar en otras variables para poder establecer un modelo causal que realmente refleje la influencia de todos los aspectos que condicionan los resultados académicos de los estudiantes de secundaria, máxime cuando los enfoques de enseñanza del profesor condicionan los de los estudiantes (Mirete, 2014).

\section{REFERENCIAS}

Balzano, S. (2002). Las construcciones culturales sobre el éxito y el fracaso escolar y sus implicaciones sobre los modelos educativos en la Argentina. Cultura y Educación, 3 (14), 283-296. Recuperado el 20 de mayo de 2012, de http://www.fia.es/revistas/culturayeducacion/home

Biggs, J.B. (1978). Individual and group differences in study processes. British Journal of Educational Psychology, 48, 266-279.

Biggs, J.B. (1987). Student approaches to learning and studying. Melburne: Australian Council for Educational Research.

Calero, J., Choi, A. \& Waisgrais, S. (2009). Determinantes del riesgo de fracaso escolar en España: una aproximación a través de un análisis logístico multinivel aplicado a PISA2006. Revista de educación. Núm. Extraordinario 2010, 225-256. Recuperado el 30 de mayo de 2012, de http://www.revistaeducacion.mec.es/rezo10.htm

De La Fuente, J. \& Justicia, F. (2004). Regulación de la enseñanza para la autorregulación del aprendizaje en la Universidad. Aula abierta, 82, 161-179. Recuperado el 20 de abril de 2012, de http://www.uniovi.net/ICE/publicaciones/Aula_Abierta/ 
De Natale, M. (1990). Rendimiento escolar. En Flores, G.; D’Acais y Gutiérrez, I. Diccionario de Ciencias de la Educación. Madrid: Paulinas.

Elliot, E. S. \& Dweck, C. S. (1988). Goals: An approach to motivation and achievement.

Journal of Personality and Social Psychology, 54, 5-12. Recuperado el 15 de mayo de 2012, de http://www.apa.org/pubs/journals/psp/index.aspx

Hernández. F. (1993). Los enfoques de aprendizaje en alumnos universitarios. Un estudio empírico. Proyecto de investigación para el acceso a Cátedra.

Hernández Pina, F., García Sanz, M. P. \& Maquilón, J. J. (2005). Análisis del cuestionario de procesos de estudio-2 factores de Biggs en estudiantes universitarios españoles. Revista Fuentes, 6.

López-Vargas, O., Hederich-Martínez, C. \& Camargo-Uribe, A. (2011). Estilo cognitivo y logro académico. Educación y Educadores, 14 (1), 67-82.

Manzano, M. (2007). Estilos de aprendizaje, estrategias de lectura y su relación con el rendimiento académico en la segunda lengua. Granada: Universidad de Granada.

Maquilón, J. J. (2001). Los enfoques de aprendizaje en estudiantes de Pedagogía. Tesis de Licenciatura. Murcia: Universidad de Murcia.

Maquilón, J. J. (2010). Diseño y evaluación del diseño de un programa de intervención para la mejora de las habilidades de aprendizaje de los estudiantes universitarios. Murcia: Editum.

Maquilón, J. J. \& Hernández Pina, F. (2011). Influencia de la motivación en el rendimiento académico de los estudiantes de formación profesional. Revista Electrónica Interuniversitaria de Formación del Profesorado, 14(1), 84-90.

Marchesi Ullastres, A. (2003). El fracaso escolar en España. Madrid: Fundación Alternativas.

Marchesi, A. \& Pérez, E. (2003). La comprensión del fracaso escolar. En A. Marchesi y C. Hernández, (Eds). El fracaso escolar. Una perspectiva internacional. Madrid: Alianza Editorial.

Mirete, A. B. (2014). TIC y enfoques de enseñanza y aprendizaje en Educación Superior. (Tesis Doctoral). Universidad de Murcia, Murcia. Recuperado de: https://digitum.um.es/jspui/bitstream/10201/40440/1/Ana\%20Mirete\%20Ru\%C3\%ADzTesis.pdf

Pérez-Díaz, V., Rodríguez, J. C. \& Sánchez, L. (2001). La familia española ante la educación de los hijos. Barcelona, Fundación La Caixa. 


\section{AUTORES}

Ana Belén Mirete Ruiz

Licenciada en Pedagogía, Máster en Psicología de la Educación y Doctora en Psicología por la Universidad de Murcia. Profesora del Departamento de Didáctica y Organización Escolar en la subárea de Inclusión Educativa. Sus líneas de investigación están orientadas hacia la innovación educativa, las inclusión educativa, el uso didáctico de las TIC como herramientas mejora de la calidad del aprendizaje y la docencia universitaria. Ha participado en varios proyectos de investigación e innovación educativa, y tiene diferentes publicaciones sobre estas y otras temáticas.

\section{Marta Soro Bernal}

Licenciada en Turismo por la Universidad de Murcia y Máster en Formación del Profesorado por la misma Universidad. Ha presentado comunicaciones sobre esta temática a congresos internacionales y participa en la línea de investigación sobre enfoques de aprendizaje y enseñanza y su relación con el fracaso escolar.

Javier J. Maquilón Sánchez

Profesor Contratado Doctor del Departamento de Métodos de Investigación y Diagnóstico en Educación de la Universidad de Murcia (España). Coordinador del Máster de Investigación e Innovación en Educación Infantil y Educación Primaria. Sus principales líneas de investigación se centran en la identificación de los enfoques de enseñanza y aprendizaje en los niveles de educación primaria, secundaria y universidad, el uso malintencionado de las tecnologías (cyberbullying) y la aplicación de la Realidad Aumentada a la mejora de la calidad de la enseñanza y el aprendizaje. Ha participado y coordinado diversos proyectos de investigación e innovación educativa orientados al EEES, impartido ponencias, cursos de formación de profesorado, presentado comunicaciones y publicado numerosos trabajos entre artículos, libros y capítulos de libro sobre ésta y otras temáticas. 